\title{
Experimental Characterization of Electrical Discharge Machining of Aluminum 6061 T6 Alloy using Different Dielectrics
}

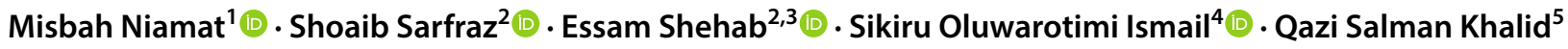

Received: 6 December 2018 / Accepted: 19 June 2019 / Published online: 8 July 2019

(c) The Author(s) 2019

\begin{abstract}
Electrical discharge machining is a non-traditional machining method broadly employed in industries for machining of parts that have typical profiles and require great accuracy. This paper investigates the effects of electrical parameters: pulse-on-time and current on three performance measures (material removal rate, microstructures and electrode wear rate), using distilled water and kerosene as dielectrics. A comparison between dielectrics for the machining of aluminum $6061 \mathrm{~T} 6$ alloy material in terms of performance measures was performed. Aluminum 6061 T6 alloy material was selected, because of its growing use in the automotive and aerospace industrial sectors. The experimental sequence was designed using Taguchi technique of $\mathrm{L}_{9}$ orthogonal array by changing three levels of pulse-on-time and current, and test runs were performed separately for each dielectric. The results obtained show that greater electrode wear rate (EWR) and higher material removal rate (MRR) were achieved with distilled water when compared with kerosene. These greater EWR and MRR responses can be attributed to the early breakage of the weak oxide and carbide layers formed on the tool and alloy material surfaces, respectively. The innovative contributions of this study include, but are not limited to, the possibility of machining of aluminum $6061 \mathrm{~T} 6$ alloy with graphite electrode to enhance machinability and fast cutting rate employing two different dielectrics.
\end{abstract}

Keywords Electric discharge machining $\cdot$ Dielectric $\cdot$ Taguchi technique $\cdot$ Electrode wear rate $\cdot$ Material removal rate . Microstructure

Shoaib Sarfraz

shoaib.sarfraz@cranfield.ac.uk

1 Mechanical Engineering Department, Muhammad Nawaz Sharif University of Engineering and Technology, Multan, Pakistan

2 Manufacturing Department, School of Aerospace, Transport and Manufacturing, Cranfield University, Cranfield MK43 0AL, Bedfordshire, UK

3 School of Engineering, Nazarbayev University, Astana, Kazakhstan

4 School of Engineering and Computer Science, University of Hertfordshire, College Lane Campus, Hatfield AL10 9AB, England, UK

5 Department of Industrial Engineering, University of Engineering and Technology Peshawar, Peshawar, Pakistan

\section{Introduction}

Electrical discharge machining (EDM) is one of the earliest non-traditional machining processes and is used for the machining of complex shapes and 3D profiles with great precision and accuracy. It is also called as a spark erosion process, where a series of rapidly occurring discharges between two current-carrying electrodes in the presence of a dielectric medium removes material from the workpiece. The required shape is achieved as a negative image imparting of the electrode. There is no direct contact between these two electrodes which makes this process free from mechanical stresses and chattering. EDM has distinctive advantages for machining surgical components, dies and mold-making, and in automobile and aerospace industries [1-4].

Being a complex dynamic machining method, a small variation in parameters may vary the responses such as surface finish, material removal rate, tool wear rate, kerf width, recast layer, among other factors to a greater extent. Process parameters selection is important in the EDM process and care must be taken during selection; otherwise, 
the productivity and quality of parts may be damaged [5]. Furthermore, it is today's foremost industrial requirement to produce fine-quality parts at a higher production rate, but also controlling the process cost [6]. Hence, explicated study is required to understand parameters and their responses to fully apply the EDM process effectively. Although different electrical parameters such as pulse-on-time, pulse-off-time, current and others are imperative, non-electrical parameters such as tool material, workpiece material and most importantly dielectrics play a decisive role in the EDM process. Dielectrics are very significant in EDM technology as they circulate between the electrode and the workpiece and are responsible for discharge phenomena which brings productivity and quality [7]. Firstly, dielectrics serve as an insulating medium, and during the process it acts as an ionization medium, and after machining it is responsible for washing of the chips and cooling of the working area [7-10]. Different dielectrics have dissimilar compositions and cooling rates; hence, the selection of a suitable dielectric is essential [11, 12]. Water, hydrocarbon oils and gases are major categories of dielectrics [8-10]. Hydrocarbon oils especially kerosene are well known in EDM, but other dielectrics need to be evaluated in order to make the EDM process more viable.

Many researchers had experimented with various parameters to investigate different performance measures during EDM. Some of them have also studied dielectric impact. Wang et al. [13] compared compound dielectric, kerosene and distilled water for machining TC4 titanium alloy. Compound dielectric resulted in highest material removal rate, lower relative electrode wear rate and smaller surface roughness values. Tang et al. [14] observed that tap water gave higher MRR due to the absence of a carbide layer in the discharge phenomena for Ti-6Al-4V alloy. Zhang et al. [15] investigated five different types of dielectrics while machining steel 8407. They reported that liquid dielectrics exhibited better material removal efficiency, while water-oil emulsion sustained pressure for a longer time. Valaki and Rathod [16] analyzed bio-dielectrics developed from vegetable oil and kerosene while machining P20 steel, the bio-dielectrics showed better results than kerosene for MRR and EWR. Furthermore, EDM process using water oil emulsion showed that both MRR and surface roughness (SR) were influenced greatly by current. Moreover, results were similar compared to that of kerosene, which confirmed the feasibility of water-oil emulsion to be used as dielectrics, as reported by Liu et al. [17] and Zhang et al. [18]. Wu et al. [19] performed a comparative study of machining in pure kerosene and kerosene with addition of powder and surfactant; $60 \%$ improved surface quality was obtained with powdered mixed dielectric. Kursad [20] performed an experimental study of hole drilling using kerosene, distilled water and water as dielectrics by changing the current level. Increasing current decreased surface quality and increased electrode wear rate, while distilled water offered better results. Baseri and Sadeghian [21] showed through experiments that EDM performance could be enhanced by adding powder in a dielectric in combination with a rotary tool, and higher current and pulse duration led to higher MRR, EWR and SR. Tebin et al. [22] evaluated parametric evaluation for steel $50 \mathrm{CrV} 4$. Higher energy density produced rougher surface due to more melting, and it also caused arcing phenomena which was an abnormal discharge. Guo et al. [23] presented optimization model of parameters for machining insulating zirconia 3 YSZ. Increasing current and/or pulse-on-time caused an increase in both MRR and EWR. Ou and Wang [24] investigated EDM machining, using water and suspension of particles in water for medical applications. Although MRR was lower in suspension-based dielectrics, however, it resulted in good surface quality. Muthuramalingam and Mohan [25] found that SR and MRR directly depended on current and duty factor, and with uniform distribution of discharge current, surface roughness was reduced. Increasing current and pulse-on-time resulted in higher MRR, while EWR increased at higher current EDM on AISI D6 tool steel, as reported by Barenji et al. [26]. Koteswararao et al. [27] performed machining of EN31 and reported that MRR decreased with elevated values of pulse duration and continue to increase with an increase in current levels. Amorim and Weingaertner [28] compared copper and graphite electrode for machining AISI P20, and graphite resulted in better surface roughness and MRR, while copper electrode offered stable discharging. Moreover, positive polarity tool offered better MRR, while good surface was obtained using negative polarity tool.

It is evident from the literature review that the selection of a proper dielectric is indispensable for the efficiency and stability of the EDM process. Dielectrics are not only providing medium for discharging, but also control the mechanism of the process. Different surface characteristics can be obtained using different dielectrics. Many researchers have compared different dielectrics for steels, titanium alloys and other engineering materials. However, little or no studies have been reported on aluminum alloy with different dielectric media. Moreover, all the above-mentioned researchers utilized copper electrodes. Hence, in this present work, the performances of distilled water and kerosene are compared. Graphite electrode was used during machining of aluminum $6061 \mathrm{~T} 6$ alloy. The aluminum was machined as the workpiece for the experimental study. In addition, the effects of process parameters: pulse-ontime and current, are also evaluated, using surface plots and line graphs to compare both dielectrics in order to select better dielectric. Furthermore, a comparison was performed on scanning electron microscope (SEM) micrographs to evaluate the effects of both change of parameters and dielectrics. 
Fig. 1 NEUAR EDM machine

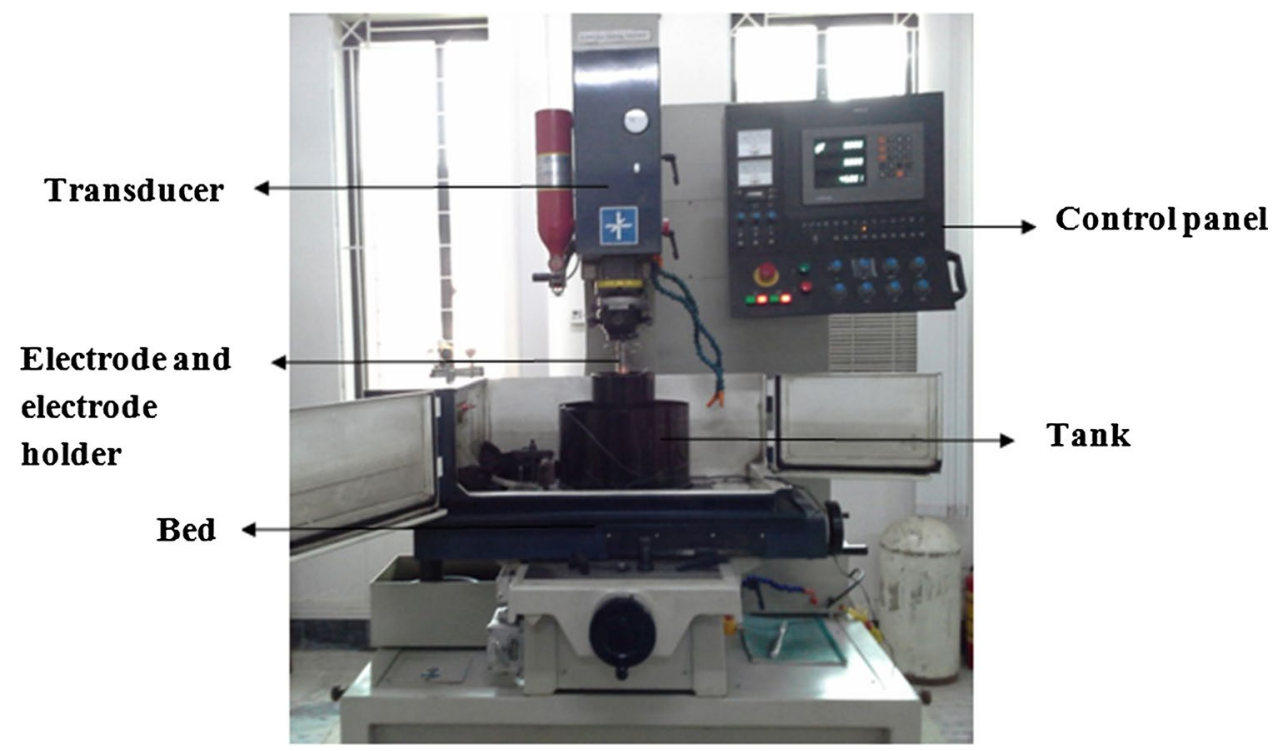

Table 1 Chemical composition (wt\%) of the workpiece (Al

\begin{tabular}{lllllll}
\hline $\mathrm{Cu}$ & $\mathrm{Ti}$ & $\mathrm{Mg}$ & $\mathrm{Fe}$ & $\mathrm{Zn}$ & $\mathrm{Si}$ & $\mathrm{Cr}$ \\
\hline 0.8 & 0.12 & 1.1 & 0.5 & 0.2 & 1.4 & 0.33 \\
\hline
\end{tabular}

Fig. 2 Dimensions of the aluminum 6061 T6 alloy used
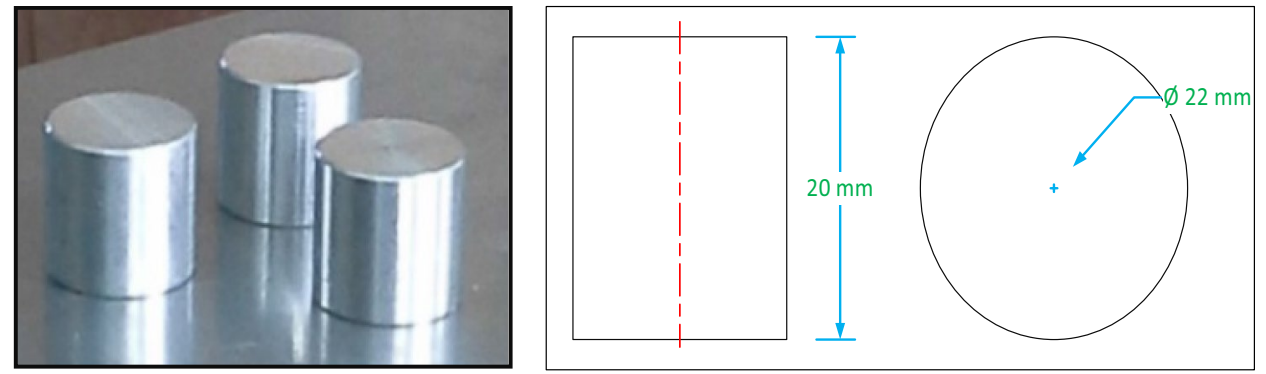

Fig. 3 Dimensions of the graphite electrode
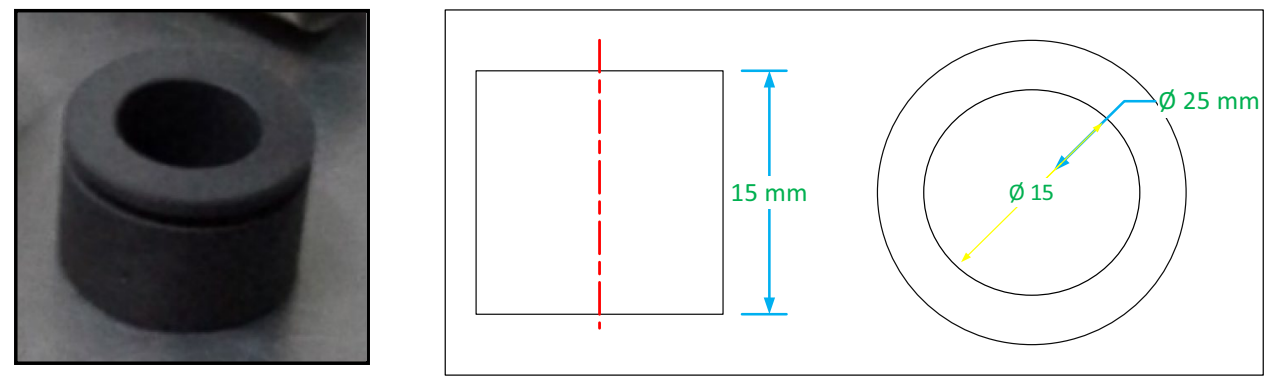

\section{Experimental Procedure}

Experiments were performed on NEUAR Diesinker EDM with the jet flushing method as depicted in Fig. 1. Aluminum $6061 \mathrm{~T} 6$ alloy was used as a workpiece. The chemical composition of the material is shown in Table 1. The materials have equal dimensions and sizes. The diameter and length were 22 and $20 \mathrm{~mm}$, respectively, as shown in Fig. 2.

A hollow cylindrical graphite electrode with external and internal diameters of 25 and $15 \mathrm{~mm}$, respectively, were used (Fig. 3). The workpiece and electrode were attached to positive and negative polarities, respectively. Taguchi technique 
Table 2 Levels of the process parameters

\begin{tabular}{lccc}
\hline Parameters & Low & Medium & High \\
\hline Pulse-on-time $(\mu \mathrm{s})$ & 60 & 90 & 120 \\
Current (ampere) & 6 & 9 & 12 \\
\hline
\end{tabular}

Table 3 Levels of the constant parameters

was used for experimental design (planning and implementation). It has a distinct advantage of offering a fewer number of experimental runs [29].

An L9 orthogonal array was employed for the design of the experimental sequence with three levels of parameters: Pulse-on-time (Pon) and current (Table 2). Process parameters (Current and Pon) have been selected based on their significant importance in EDM [23-27]. The correct selection of the ranges of these parameters was based on literature review and preliminary trials in such a way that the machined parts were defect free. Some parameters were set at a constant level as shown in Table 3 . The experimental or test runs are provided in Table 4. Firstly, a separate analysis of electrical parameters (pulse-on-time and current) was studied for EWR and MRR using distilled water and kerosene; then, MRR and EWR were compared for both dielectrics.

A separate electrode and workpiece were used for each experimental run. The weights of the workpiece and electrode were measured using a weight balance before and after each experiment. Machining time was recorded using a stopwatch. After each run, all the workpieces and electrodes were cleaned with acetone to remove dielectric remains. For the calculations of the MRR and EWR, the following equations were employed [30]:

$\operatorname{MRR}=\frac{\text { Volume of the work material removed }}{\text { Machining time }}=\frac{W_{b}-W_{a}}{\rho \times T_{m}}$

EWR $=\frac{\text { Volume of the tool material removed }}{\text { Machining time }}=\frac{E_{b}-E_{a}}{\rho \times T_{m}}$

where $W_{a}$ and $W_{b}$ represent the weight of the workpieces before and after machining for each experiment; $E_{b}$ and $E_{a}$ denote weight values of electrode prior and subsequent to the experiment, $\rho$ denotes density; and $T_{m}$ represents time utilized for machining. After computing MRR and EWR values, signal-to-noise ratios $(\mathrm{S} / \mathrm{N})$ were estimated to study precisely the trend of parameters. Similarly, Taguchi approach was used to measure $\mathrm{S} / \mathrm{N}$ ratios, using larger the better trend for MRR and smaller the better trend for EWR. These two expressions are shown as Eqs. (3) and (4), as adopted from [31]:

Larger the better $\left(\frac{S}{N}\right)=-\log \left(\frac{1}{n} \sum\left(\frac{1}{y^{2}}\right)\right)$

Smaller the better $\left(\frac{S}{N}\right)=-\log \left(\frac{1}{n} \sum\left(y^{2}\right)\right)$

where $y$ represents values obtained after experiments for both MRR and EWR in each experiment and $n$ indicates the number of experiments.

For micrographs, samples were prepared by first dipping them into hardener and resin as depicted in Fig. 4; then, dried to stabilize them. TESCAN (MIRA 3 XMU

Table 4 Design matrix with response values

\begin{tabular}{|c|c|c|c|c|c|c|c|c|c|c|}
\hline \multirow[t]{2}{*}{ Run no. } & \multirow[t]{2}{*}{ Current (A) } & \multirow[t]{2}{*}{ Pon $(\mu \mathrm{s})$} & \multicolumn{2}{|c|}{$\operatorname{MRR}\left(\mathrm{mm}^{3} / \mathrm{min}\right)$} & \multicolumn{2}{|c|}{$\operatorname{EWR}\left(\mathrm{mm}^{3} / \mathrm{min}\right)$} & \multicolumn{2}{|c|}{$\mathrm{S} / \mathrm{N}$ for $\mathrm{MRR}(\mathrm{dB})$} & \multicolumn{2}{|c|}{$\mathrm{S} / \mathrm{N}$ for $\mathrm{EWR}(\mathrm{dB})$} \\
\hline & & & Distilled water & Kerosene & Distilled water & Kerosene & Distilled water & Kerosene & Distilled water & Kerosene \\
\hline 1 & 6 & 60 & 11.11 & 1.70 & 0.60 & 0.55 & 20.9143 & 4.6090 & -3.6938 & 5.1927 \\
\hline 2 & 6 & 90 & 14.29 & 20.13 & 0.52 & 0.68 & 23.1006 & 26.076 & -5.8893 & 3.3498 \\
\hline 3 & 6 & 120 & 12.04 & 24.40 & 0.86 & 0.68 & 21.6125 & 27.748 & -7.6042 & 3.3498 \\
\hline 4 & 9 & 60 & 22.84 & 14.60 & 2.06 & 0.47 & 27.1739 & 23.287 & -6.1926 & 6.5580 \\
\hline 5 & 9 & 90 & 25.18 & 26.70 & 1.72 & 1.43 & 28.0228 & 28.530 & -9.1273 & -3.1067 \\
\hline 6 & 9 & 120 & 32.76 & 31.80 & 2.10 & 0.87 & 30.3069 & 30.049 & -10.3703 & 1.2096 \\
\hline 7 & 12 & 60 & 36.11 & 26.43 & 4.13 & 2.30 & 31.1525 & 28.442 & -7.2346 & -7.2346 \\
\hline 8 & 12 & 90 & 51.85 & 33.07 & 4.75 & 1.77 & 34.2950 & 30.389 & -9.7428 & -4.9595 \\
\hline 9 & 12 & 120 & 49.50 & 53.09 & 3.73 & 0.97 & 33.8921 & 34.500 & -9.7428 & 0.2645 \\
\hline
\end{tabular}




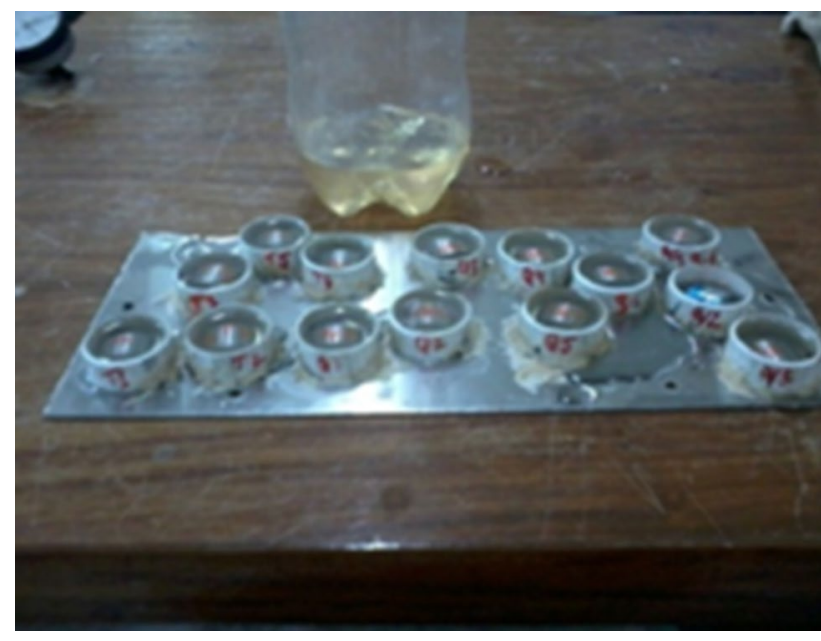

Fig. 4 Specimens made in resin

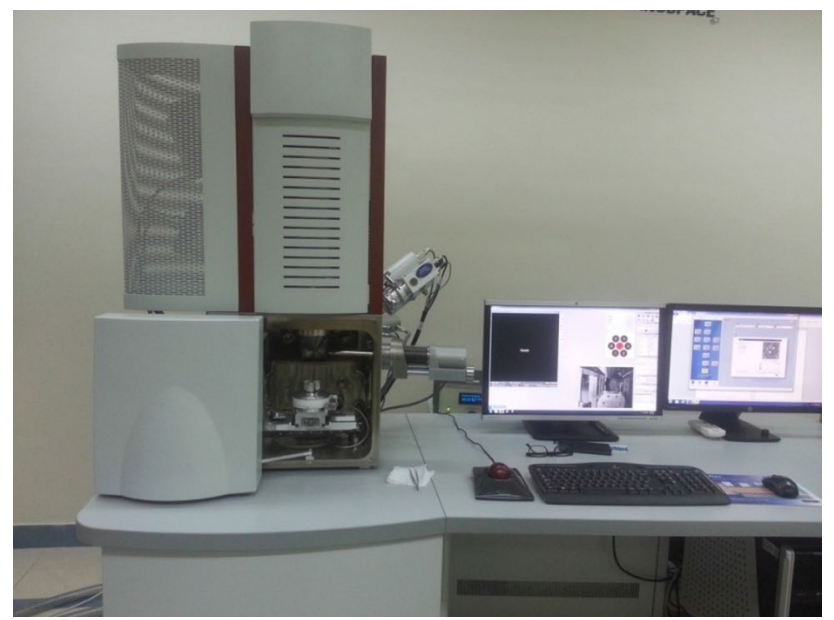

Fig. 5 Scanning electron microscope (TESCAN MIRA 3 XMU)

type) scanning electron microscope (Fig. 5) was used to obtain clear micrographs.

\section{Results and Discussion}

\subsection{Mathematical Model Development}

Regression analysis has been performed for the modeling of response variables using commercial statistical software (Minitab). Analysis of variance (ANOVA) was employed to check the relative importance of process variables with respect to responses and the adequacy of the developed models.

The ANOVA results obtained comprise of significant terms ( $p$ values less than 0.05 ) along with adequacy measure $R^{2}$ and adjusted $R^{2}$ as depicted in Table 5. The values of
Table 5 ANOVA results of MRR and EWR for kerosene and distilled water

\begin{tabular}{|c|c|c|c|c|c|}
\hline Source & Sum of squares & $d f$ & Mean square & $F$ value & $p$ value \\
\hline \multicolumn{6}{|c|}{ For $M R R_{\text {kerosene }}$} \\
\hline Current & 3057.62 & 2 & 1528.81 & 50.26 & 0.001 \\
\hline Pon & 180.05 & 2 & 90.03 & 2.96 & 0.163 \\
\hline Error & 121.67 & 4 & 30.42 & & \\
\hline Total & 3359.35 & 8 & & & \\
\hline$R^{2}$ & 0.964 & & Adj. $R^{2}$ & 0.928 & \\
\hline \multicolumn{6}{|c|}{ For $M R R_{\text {dist water }}$} \\
\hline Current & 116.51 & 2 & 58.26 & 2.69 & 0.182 \\
\hline Pon & 1677.21 & 2 & 838.60 & 38.65 & 0.002 \\
\hline Error & 86.78 & 4 & 21.70 & & \\
\hline Total & 1880.50 & 8 & & & \\
\hline$R^{2}$ & 0.954 & & Adj. $R^{2}$ & 0.908 & \\
\hline \multicolumn{6}{|c|}{ For EWR $R_{\text {kerosene }}$} \\
\hline Current & 1.58909 & 2 & 0.79454 & 39.48 & 0.002 \\
\hline Pon & 1.39509 & 2 & 0.69754 & 34.66 & 0.003 \\
\hline Error & 0.08051 & 4 & 0.02013 & & \\
\hline Total & 3.06469 & 8 & & & \\
\hline$R^{2}$ & 0.974 & & Adj. $R^{2}$ & 0.948 & \\
\hline \multicolumn{6}{|c|}{ For $M R R_{\text {dist water }}$} \\
\hline Current & 1.47642 & 2 & 0.73821 & 25.64 & 0.005 \\
\hline Pon & 1.31102 & 2 & 0.65551 & 22.77 & 0.007 \\
\hline Error & 0.11518 & 4 & 0.02879 & & \\
\hline Total & 2.90262 & 8 & & & \\
\hline$R^{2}$ & 0.963 & & $\operatorname{Adj} . R^{2}$ & 0.921 & \\
\hline
\end{tabular}

adequacy measure $R^{2}$ and adjusted $R^{2}$ are approximately 1 , which indicates an adequate signal. Therefore, the developed models can be used to navigate the design space. The final empirical models for responses of $\mathrm{MRR}_{\text {kerosene }}, \mathrm{MRR}_{\text {dist water, }}$, $\mathrm{EWR}_{\text {kerosene }}$ and EWR $\mathrm{R}_{\text {dist water }}$ are presented in Eqs. 5-8.

$\mathrm{MRR}_{\text {kerosene }}=-33.5+7.52$ Current $(A)+0.181$ Pon $(\mu \mathrm{s})$

$\mathrm{MRR}_{\text {dist water }}=-33.7+5.56$ Current $(A)+0.135$ Pon $(\mu \mathrm{s})$

$\mathrm{EWR}_{\text {kerosene }}=0.484+0.14$ Current $(A)+0.0161$ Pon $(\mu \mathrm{s})$

$\mathrm{EWR}_{\text {dist water }}=-0.216+0.138$ Current $(A)+0.0159$ Pon $(\mu \mathrm{s})$

\subsection{Analysis of MRR}

Normal probability plotted for resultant MRR values for kerosene is presented in Fig. 6a. All values lie on a straight line, which depicts a normal trend in error distribution. Figure $6 \mathrm{~b}$ shows the three-dimensional surface plot for MRR in relation to current and Pon. MRR increases gradually as the 


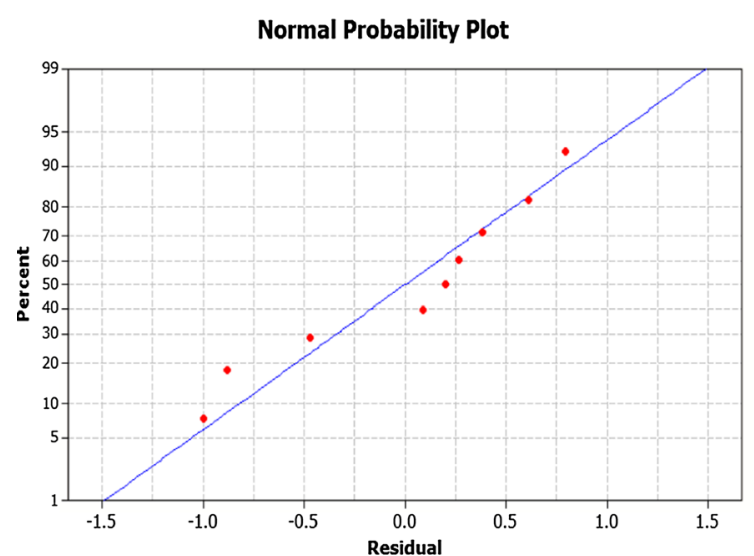

(a)

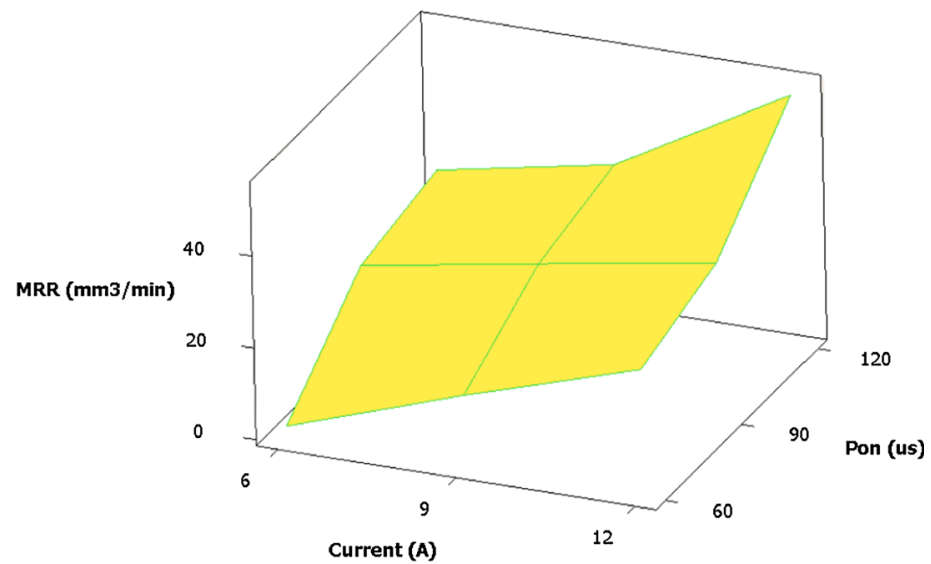

(b)

Fig. 6 a Normal plot of residuals and $\mathbf{b}$ 3D response surface plot of MRR for kerosene

value of current increases, showing a direct relation at lower values of Pon which is $60 \mu$ s, while a significant increase in MRR is observed at a higher level of Pon of $120 \mu$ s. This behavior is due to the fact that current and Pon with increasing levels exhibit more energy density for material removal and a maximum MRR of $53.09 \mathrm{~mm}^{3} / \mathrm{min}$ is observed at a current of $12 \mathrm{~A}$ and Pon of $120 \mu \mathrm{s}$. Similar trend of results has been observed from other studies [17-20].

Normal probability plot using distilled water shows that the MRR data points are normally distributed, which is illustrated in Fig. 7a. The surface plot for MRR in distilled water (Fig. 7b) shows that MRR increases with an increment in current levels from 6 to 12 amperes. Maximum MRR results occur at $51.85 \mathrm{~mm}^{3} / \mathrm{min}$, at a current of 12 amperes and Pon of $90 \mu \mathrm{s}$. For Pon, MRR increases with an increasing parameter value; then, it decreases showing a varying effect. In distilled water, an oxide layer is created on the surface of the workpiece, which on further discharging decomposes rapidly and arcing results in the non-valuable removal of material. These phenomena have been similarly reported by [22].

A comparison of MRR values for kerosene and distilled water against experimental runs or coupons is plotted as a time series plot in Fig. 8. It is evident from Fig. 8 that in some of the experimental runs (2nd, between 4th and 6th) MRR is almost identical for both dielectrics and in some cases (mainly 3rd and 9th runs); it is higher when using kerosene. Overall, MRR in distilled water is higher than kerosene. Moreover, the material removal mechanism is steadier in distilled water because of its stable discharging, while for kerosene decomposed carbon content packs on the electrode surface hinders further discharging phenomena [22]. However, in a few cases the trend is found to be reversed. This is due to the fact that when Pon is increased for same value of current, arcing occurs and leads to a decrease in the MRR

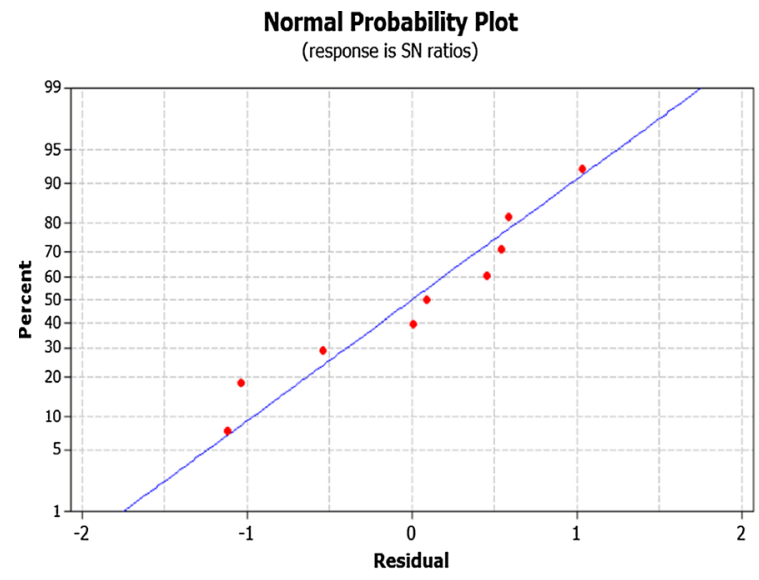

(a)

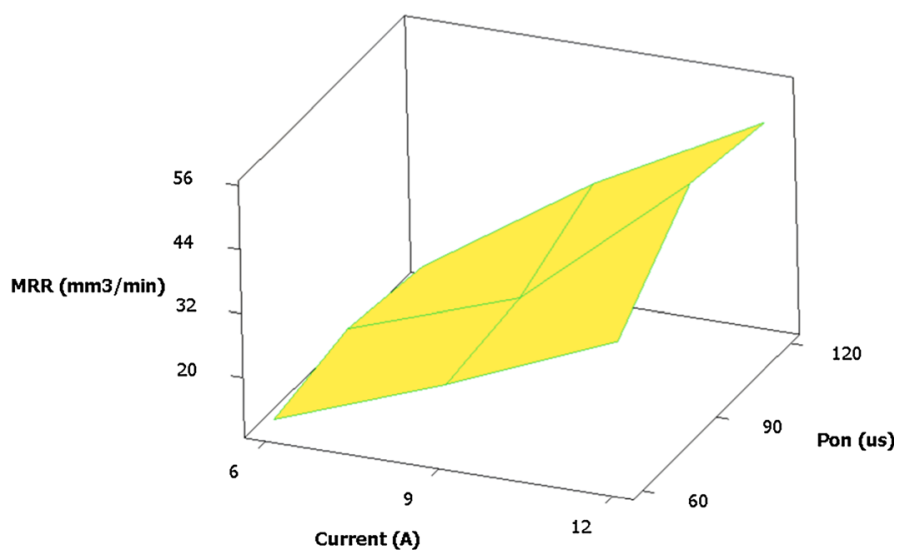

(b)

Fig. 7 a Normal plot of residuals and $\mathbf{b}$ 3D response surface plot of MRR for distilled water for distilled water 


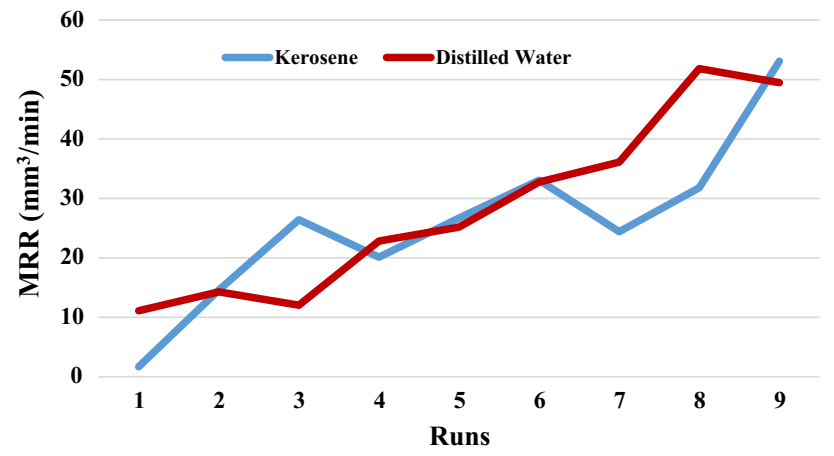

Fig. 8 Comparison of MRR using kerosene and distilled water

using distilled water. Arcing is a detrimental phenomenon and caused by a large energy discharge, which is an abnormal occurrence in electrical discharge machining [24].

\subsection{Analysis of EWR}

Figure $9 \mathrm{a}$ and $\mathrm{b}$ demonstrates the normal plot of residuals and 3D response plot for EWR, respectively. It is evident that all values lie on a straight line which shows that anticipated values are in good agreement with the actual values. EWR slowly increases up to 9 amperes current followed by a rapid increase (for 12 amperes). This implies that at a high current more wear of the electrode occurs as supported by [27]. As Pon increases from 60 to $90 \mu$ s, the wear rate increases and then starts decreasing up to $120 \mu \mathrm{s}$; it decreases even at higher values of current because decomposed carbide layers stick on the electrode surface which is hard to break and avoids further wearing of the electrode.

Figure 10a confirms that data distribution is normal. The surface plot of EWR against current and Pon shows a varying effect for current and Pon as shown in Fig. 10b. EWR increases up to a current of 9 amperes and pulse duration of $90 \mu$ s; then, it decreases. This is because layers of oxides are formed when working with distilled water. These layers break too early to produce EWR, but with a further increment to the parameter levels of Pon of $120 \mu$ s and current of 12 amperes abnormal discharging (arcing) occurs. The maximum EWR of $3.07 \mathrm{~mm}^{3} / \mathrm{min}$ is observed at a current of 9 amperes.

A comparison plot based on values of EWR for both dielectrics is presented in Fig. 11. It is evident that EWR is more dominant in distilled water than kerosene. This can be attributed to the non-decomposition of carbon formed as a carbide layer on the electrode surface which protects the electrode from further wear, with kerosene during discharge. In the case of distilled water, an oxide layer forms on the electrode surface which easily breaks off and undergoes further wear, as similarly reported by Wang et al. [13].

\subsection{Microstructure Analysis}

During machining, deep and overlapping craters are created owing to the successive intense heat, electrical discharge and local melting or vaporization of the alloy material. Micrographs of machined surfaces were observed and captured with SEM TESCAN (3 XMU, MIRA). The surfaces showed irregular topography, comprising craters of various sizes, micro-cracks, lumps of debris and spherical deposits, as depicted in Fig. 12a and b.

The surface properties might have been altered due to the changing values of the process parameters such as pulseon-time and current. A micrographic examination was performed keeping the pulse-on-time at $60 \mu$ and changes to the values of current for both kerosene and distilled water. Some of the molten material produced as a result of sparks is flushed away by the dielectric, while the remaining sticks

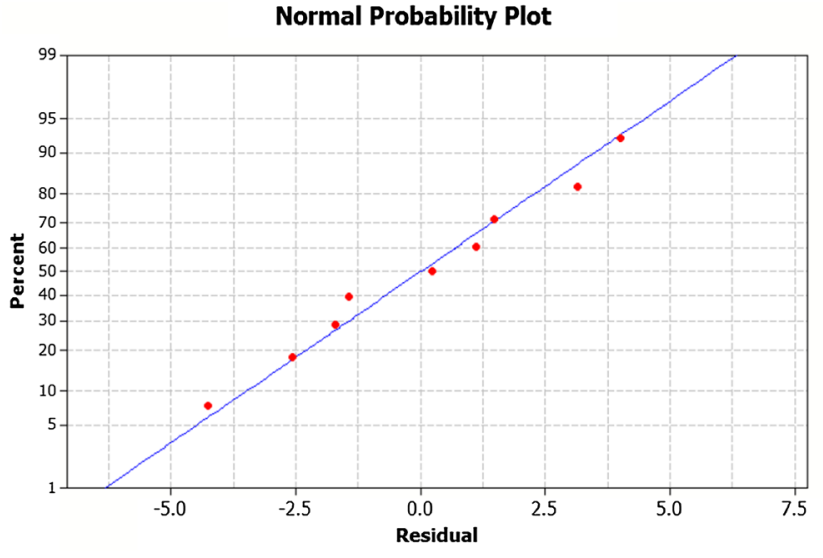

(a)

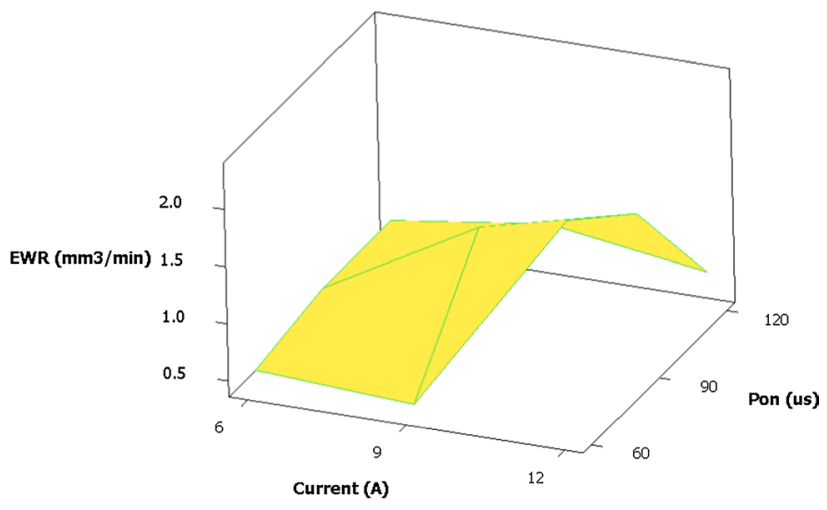

(b)

Fig. 9 a Normal plot of residuals and $\mathbf{b}$ 3D response surface plot of EWR for kerosene 


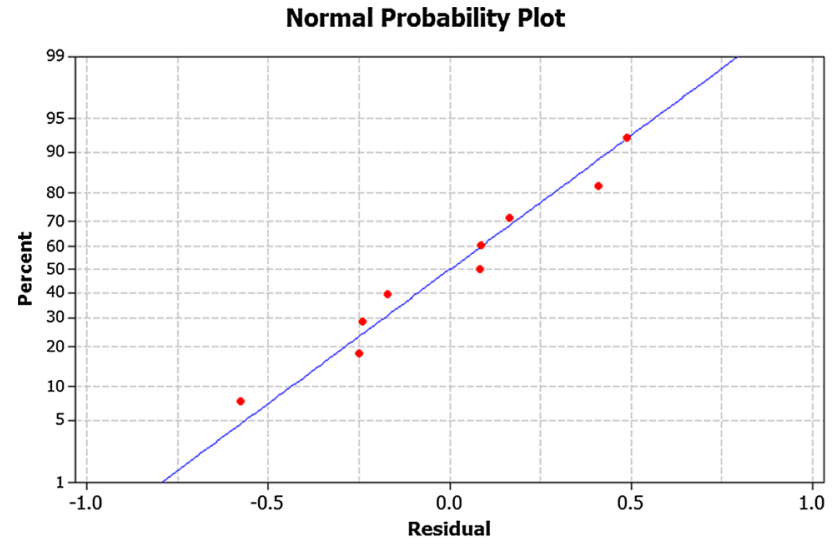

(a)

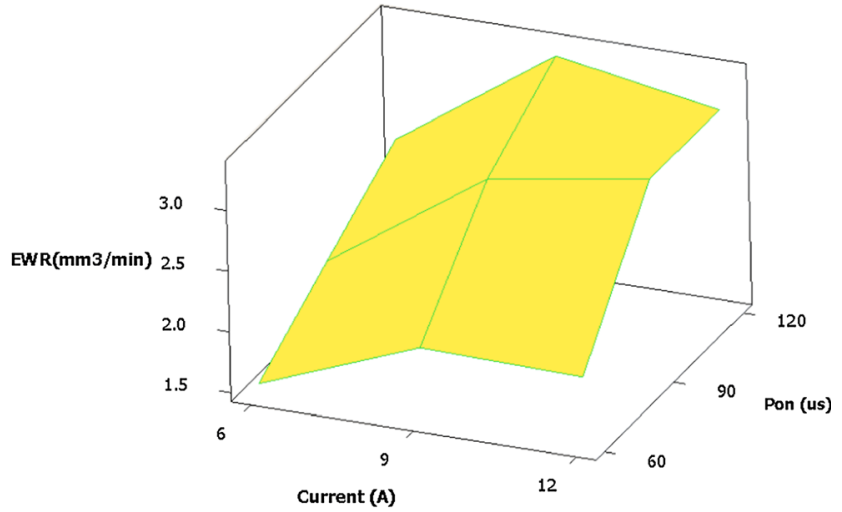

(b)

Fig. 10 a Normal plot of residuals and $\mathbf{b}$ 3D response surface plot of EWR for distilled water

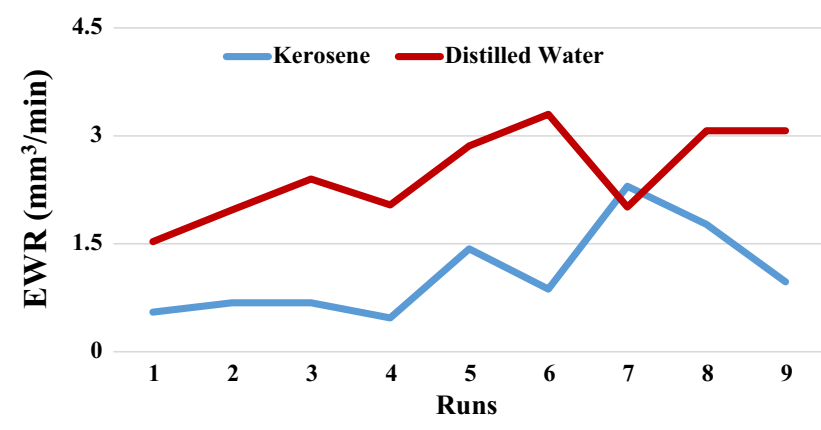

Fig. 11 Comparison of EWR using kerosene and distilled water

onto the surface as lumps of debris and spherical deposits. The degree of surface cracks and their depths are directly associated with the discharge energy; discharge energy increases the occurrence of these cracks. Comparison of the micrographs taken at 6 and 9 amperes in a kerosene dielectric are shown in Fig. 12a and b, respectively. These Figures show that more cracks and deeper craters appear as the current level is increased. Similar results were observed by increasing the current in distilled water as shown in Fig. 13a and $b$. At an elevated current, the impact of discharge energy on the surface of the workpiece becomes higher and resulting erosion causes an increase in the wear and tear (surface roughness). Additionally, comparison of Figs. 12a and 13a shows that distilled water results in a rougher surface when compared with kerosene at the same level of current of 6 amperes and pulse-on-time of $60 \mu \mathrm{s}$. Material removal in distilled water usually occurs through crack propagation and melting; therefore, more cracks and surface defects are produced. Similar results were observed at a current of 9 amperes, as illustrated in Figs. 12b and 13b.

\section{Conclusions}

In this experimental work, the influences of distilled water and kerosene dielectrics have been investigated for EDM machining of aluminum $6061 \mathrm{~T} 6$ alloy using a graphite
Fig. 12 SEM micrographs taken at Pon of $60 \mu$ s and currents of: a 6 amperes b 9 amperes for kerosene

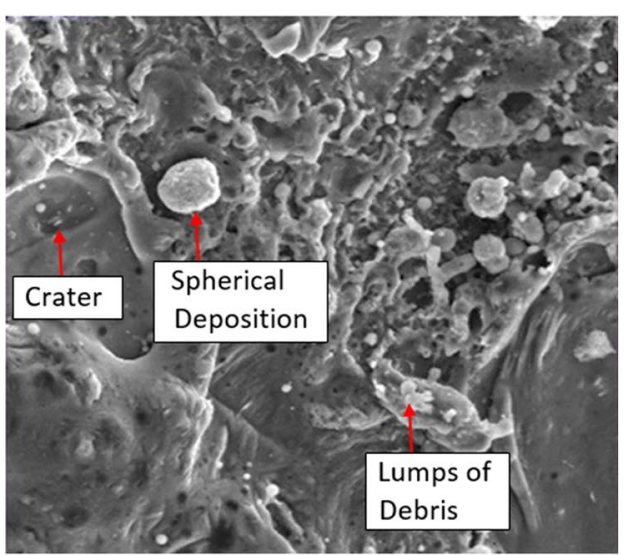

(a)

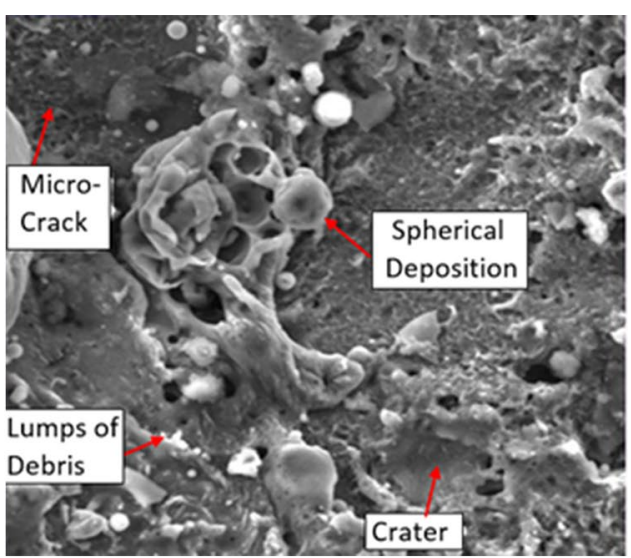

(b) 
Fig. 13 SEM micrographs taken at Pon of $60 \mu$ s and currents of: a 6 amperes b 9 amperes for distilled water

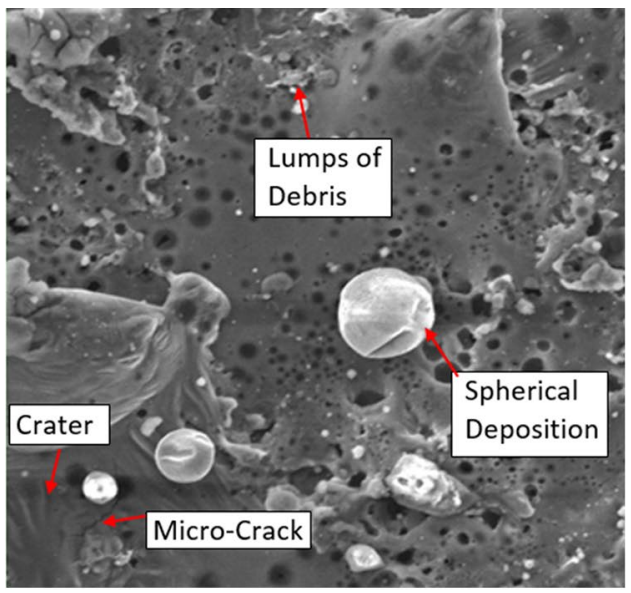

(a)

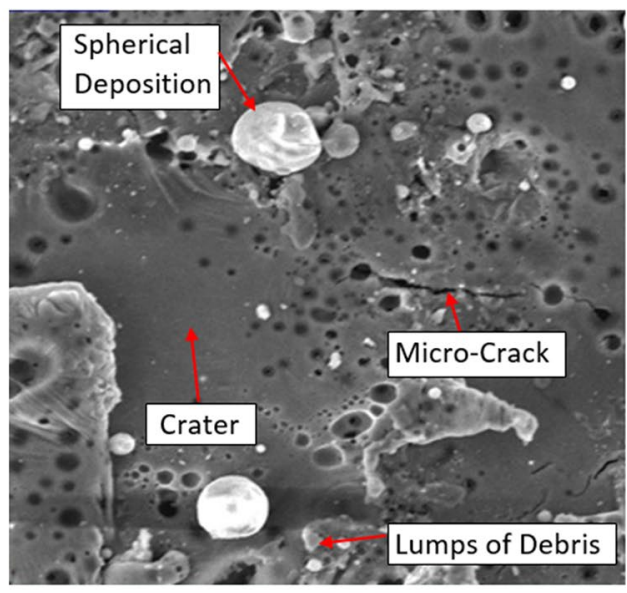

(b) electrode. Moreover, the impact of pulse-on-time and current on MRR, SR and microstructures has been evaluated separately for both dielectrics. The following important conclusions are drawn from this innovative experimental study:

- Maximum MRR is obtained at higher values of pulseon-time and current. Also, higher MRR values were achieved in distilled water compared to kerosene because of the formation of a thin carbide layer on the surface of the alloy, which further reduced the material removal phenomenon.

- Both current and pulse-on-time exhibited varying effects on EWR, as maximum EWR was obtained at higher current levels. It is interesting to note that greater EWR was recorded in distilled water compared to kerosene due to the early breakage of the weak oxide layer formed on the surface of the tool.

- Microstructure analysis revealed that higher values of current produced rougher surface, as greater discharge energy was available for the melting of the material. Therefore, poor surface was observed with distilled water as depicted in micrographs.

Evidently, EDM is an adequate process for machining aluminum 6061 T6 alloy. The results obtained from this study clearly show that distilled water has better dielectric properties in comparison with kerosene. Also, distilled water offers better MRR values in addition to being an environmentally friendly dielectric. Therefore, it should be a preferred dielectric for EDM. Moreover, graphite, as an electrode offers a better MRR with low processing cost and good thermal stability. Hence, it should be employed widely for this non-conventional machining (EDM). For future work, different aluminum alloys can be explored along with different dielectrics, such as dry EDM setup and dielectrics mixed with nanoparticles. Lastly, a study on the effects of different electrode shapes is also recommended.
Acknowledgements The authors sincerely express their appreciations to the head and staff of Fracture and Mechanics Laboratory, University of Engineering and Technology Taxila, for their support, assistance and provision of facilities to conduct this experimental study.

Open Access This article is distributed under the terms of the Creative Commons Attribution 4.0 International License (http://creativeco mmons.org/licenses/by/4.0/), which permits unrestricted use, distribution, and reproduction in any medium, provided you give appropriate credit to the original author(s) and the source, provide a link to the Creative Commons license, and indicate if changes were made.

\section{References}

1. Raut, T.G.; Shinde, M.Y.: A review on optimization of machining parameters in EDM. Int. J. Innov. Res. Sci. Eng. Technol. 4, 893-896 (2015)

2. Huang, H.; Zhang, Z.; Ming, W.; Xu, Z.; Zhang, Y.: A novel numerical predicting method of electric discharge machining process based on specific discharge energy. Int. J. Adv. Manuf. Technol. 88, 409-424 (2017). https://doi.org/10.1007/s0017 0-016-8688-Z

3. Pachaury, Y.; Tandon, P.: An overview of electric discharge machining of ceramics and ceramic based composites. J. Manuf. Process. 25, 369-390 (2017). https://doi.org/10.1016/j.jmapr o.2016.12.010

4. Jamwal, A.; Aggarwal, A.; Gautam, N.; Devarapalli, A.: Electrodischarge machining: recent developments and trends. Int. Res. J. Eng. Technol. 5, 433-448 (2018)

5. Mohanty, C.P.; Mahapatra, S.S.; Singh, M.R.: An intelligent approach to optimize the EDM process parameters using utility concept and QPSO algorithm. Eng. Sci. Technol. Int. J. 20, 552-562 (2017). https://doi.org/10.1016/j.jestch.2016.07.003

6. Sahu, S.K.; Naik, S.; Das, S.R.; Dhupal, D.: Parametric optimization of surface roughness and overcut in electric discharge machining of Al-SiC using copper electrode. In: Chattopadhyay, J., Singh, R., Prakash, O. (eds.) Renewable Energy and its Innovative Technologies, pp. 99-116. Springer, Singapore (2019)

7. Leão, F.N.; Pashby, I.R.: A review on the use of environmentally-friendly dielectric fluids in electrical discharge machining. J. Mater. Process. Technol. 149, 341-346 (2004). https://doi. org/10.1016/j.jmatprotec.2003.10.043 
8. Mohd Abbas, N.; Solomon, D.G.; Fuad Bahari, M.: A review on current research trends in electrical discharge machining (EDM). Int. J. Mach. Tools Manuf 47, 1214-1228 (2007). https://doi. org/10.1016/j.ijmachtools.2006.08.026

9. Zhang, Q.H.; Du, R.; Zhang, J.H.; Zhang, Q.B.: An investigation of ultrasonic-assisted electrical discharge machining in gas. Int. J. Mach. Tools Manuf 46, 1582-1588 (2006). https://doi. org/10.1016/j.ijmachtools.2005.09.023

10. Niamat, M.; Sarfraz, S.; Aziz, H.; Jahanzaib, M.; Shehab, E.; Ahmad, W.; Hussain, S.: Effect of different dielectrics on material removal rate, electrode wear rate and microstructures in EDM. Proc. CIRP. 60, 2-7 (2017). https://doi.org/10.1016/j.proci r.2017.02.023

11. Zhang, Y.; Liu, Y.; Ji, R.; Cai, B.: Study of the recast layer of a surface machined by sinking electrical discharge machining using water-in-oil emulsion as dielectric. Appl. Surf. Sci. 257, 5989-5997 (2011). https://doi.org/10.1016/j.apsusc.2011.01.083

12. Chakraborty, S.; Dey, V.; Ghosh, S.K.: A review on the use of dielectric fluids and their effects in electrical discharge machining characteristics. Precis. Eng. 40, 1-6 (2015). https://doi. org/10.1016/j.precisioneng.2014.11.003

13. Wang, X.; Liu, Z.; Xue, R.; Tian, Z.; Huang, Y.: Research on the influence of dielectric characteristics on the EDM of titanium alloy. Int. J. Adv. Manuf. Technol. 72, 979-987 (2014). https:// doi.org/10.1007/s00170-014-5716-8

14. Tang, L.; Du, Y.T.: Multi-objective optimization of green electrical discharge machining Ti-6Al-4 V in Tap water via GreyTaguchi method. Mater. Manuf. Process. 29, 507-513 (2014). https://doi.org/10.1080/10426914.2013.840913

15. Zhang, Y.; Liu, Y.; Shen, Y.; Ji, R.; Li, Z.; Zheng, C.: Investigation on the influence of the dielectrics on the material removal characteristics of EDM. J. Mater. Process. Technol. 214, 1052-1061 (2014). https://doi.org/10.1016/j.jmatprotec.2013.12.012

16. Valaki, J.B.; Rathod, P.P.: Investigating feasibility through performance analysis of green dielectrics for sustainable electric discharge machining. Mater. Manuf. Process. 31, 541-549 (2016). https://doi.org/10.1080/10426914.2015.1070430

17. Liu, Y.; Zhang, Y.; Ji, R.; Cai, B.; Wang, F.; Tian, X.; Dong, $\mathrm{X}$.: Experimental characterization of sinking electrical discharge machining using water in oil emulsion as dielectric. Mater. Manuf. Process. 28, 355-363 (2013). https://doi.org/10.1080/10426 914.2012.700162

18. Zhang, Y.; Liu, Y.; Ji, R.; Cai, B.; Shen, Y.: Sinking EDM in water-in-oil emulsion. Int. J. Adv. Manuf. Technol. 65, 705-716 (2013). https://doi.org/10.1007/s00170-012-4210-4

19. Wu, K.L.; Yan, B.H.; Huang, F.Y.; Chen, S.C.: Improvement of surface finish on SKD steel using electro-discharge machining with aluminum and surfactant added dielectric. Int. J. Mach. Tools Manuf. 45, 1195-1201 (2005). https://doi.org/10.1016/j.ijmac htools.2004.12.005
20. Göv, K.: Influence of coolant on the performance of electro discharge hole drilling. J. Polytech. 20, 191-196 (2017). https://doi. org/10.2339/2017.20.1191-196

21. Baseri, H.; Sadeghian, S.: Effects of nanopowder TiO2-mixed dielectric and rotary tool on EDM. Int. J. Adv. Manuf. Technol. 83, 519-528 (2016). https://doi.org/10.1007/s00170-015-7579-z

22. Tebni, W.; Boujelbene, M.; Bayraktar, E.: Parametric approach model for determining electrical discharge machining (EDM) conditions: effect of cutting parameters on the surface integrity. Adv. Mater. Res. 83-86, 725-737 (2009). https://doi.org/10.4028/ www.scientific.net/AMR.83-86.725

23. Guo, Y.; Wang, L.; Zhang, G.; Hou, P.: Multi-response optimization of the electrical discharge machining of insulating zirconia. Mater. Manuf. Process. 32, 294-301 (2017). https://doi. org/10.1080/10426914.2016.1176180

24. Ou, S.-F.; Wang, C.-Y.: Effects of bioceramic particles in dielectric of powder-mixed electrical discharge machining on machining and surface characteristics of titanium alloys. J. Mater. Process. Technol. 245, 70-79 (2017). https://doi.org/10.1016/j.jmatprotec .2017.02.018

25. Muthuramalingam, T.; Mohan, B.: Influence of discharge current pulse on machinability in electrical discharge machining. Mater. Manuf. Process. 28, 375-380 (2013). https://doi. org/10.1080/10426914.2012.746700

26. Barenji, R.V.; Pourasl, H.H.; Khojastehnezhad, V.M.: Electrical discharge machining of the AISI D6 tool steel: prediction and modeling of the material removal rate and tool wear ratio. Precis. Eng. 45, 435-444 (2016). https://doi.org/10.1016/j.precisione ng.2016.01.012

27. Koteswararao, B.; Siva Kishore Babu, K.; Ravi, D.; Kumar, K.K.; Chandra Shekar, P.: Investigation of machining parameter in EDM of high carbon steel alloy (EN31). Mater. Today Proc. 4, 13751384 (2017). https://doi.org/10.1016/j.matpr.2017.01.159

28. Amorim, F.L.; Weingaertner, W.L.: The behavior of graphite and copper electrodes on the finish die-sinking electrical discharge machining (EDM) of AISI P20 tool steel. J. Braz. Soc. Mech. Sci. Eng. 29, 366-371 (2007). https://doi.org/10.1590/S1678-58782 007000400004

29. Jung, J.H.; Kwon, W.T.: Optimization of EDM process for multiple performance characteristics using Taguchi method and grey relational analysis. J. Mech. Sci. Technol. 24, 1083-1090 (2010). https://doi.org/10.1007/s12206-010-0305-8

30. Gholipoor, A.; Baseri, H.; Shabgard, M.R.: Investigation of near dry EDM compared with wet and dry EDM processes. J. Mech. Sci. Technol. 29, 2213-2218 (2015). https://doi.org/10.1007/ s12206-015-0441-2

31. Lin, Y.-C.; Chen, Y.-F.; Wang, D.-A.; Lee, H.-S.: Optimization of machining parameters in magnetic force assisted EDM based on Taguchi method. J. Mater. Process. Technol. 209, 3374-3383 (2009). https://doi.org/10.1016/j.jmatprotec.2008.07.052 